\title{
Mycobacterium tuberculosis complex CRISPR genotyping: improving efficiency, throughput and discriminative power of 'spoligotyping' with new spacers and a microbead-based hybridization
} assay

\author{
Correspondence \\ Christophe Sola \\ christophe.sola@u-psud.fr
}

Received 21 October 2009

Accepted 26 November 2009

\begin{abstract}
Jian Zhang, ${ }^{1}$ Edgar Abadia, ${ }^{1}$ Guislaine Refregier, ${ }^{1}$ Silva Tafaj, ${ }^{2}$ Maria Laura Boschiroli, ${ }^{3}$ Bertrand Guillard, ${ }^{4}$ Antoine Andremont, ${ }^{5}$ Raymond Ruimy ${ }^{5}$ and Christophe Sola ${ }^{1,6}$
\end{abstract}

\author{
${ }^{1}$ IGEPE Team, Institute of Genetics and Microbiology, UMR8621, Universud, CNRS Université \\ Paris-Sud 11, Campus d'Orsay, F-91405 Orsay-Cedex, France \\ ${ }^{2}$ National TB Reference Laboratory, University Hospital of Lung Diseases 'Shefqet Ndroqi', \\ Tirana, Albania \\ ${ }^{3}$ Agence Française de Sécurité Sanitaire des Aliments, Maisons-Alfort, France \\ ${ }^{4}$ Institut Pasteur du Cambodge, Pnom-Penh, Cambodia \\ ${ }^{5}$ Microbiology Laboratory, Bichat-Claude Bernard Hospital, Paris, France \\ ${ }^{6}$ Unité de Génétique Mycobactérienne, Institut Pasteur, Paris, France
}

\begin{abstract}
The aims of the present study were to implement a microbead-based 'spoligotyping' technique and to evaluate improvements by the addition of a panel of 25 extra spacers that we expected to provide an increased resolution on principal genetic group 1 (PGG 1) strains. We confirmed the high sensitivity and reproducibility of the classical technique using the 43 spacer panel and we obtained perfect agreement between the membrane-based and the microbead-based techniques. We further demonstrated an increase in the discriminative power of an extended 68 spacer format for differentiation of PGG 1 clinical isolates, in particular for the East AfricanIndian clade. Finally, we define a limited yet highly informative reduced 10 spacer panel set which could offer a more cost-effective option for implementation in resource-limited countries and that could decrease the need for additional VNTR (variable number of tandem repeats) genotyping work in molecular epidemiological studies. We also present an economic analysis comparing membrane-based and microbead-based techniques.
\end{abstract}

\section{INTRODUCTION}

The current global plan for tuberculosis (TB) control suggests that it will be difficult to eradicate the disease by 2050 (Dye \& Williams, 2008). This issue is particularly

\footnotetext{
Abbreviations: CAS, Central Asian; CRISPR, clustered regularly interspaced palindromic repeats; DR, direct repeat; EAI, East African-Indian; EDC, $N$-(3-dimethylaminopropyl)-N'-ethylcarbodiimide; LAM, LatinAmerican and Mediterranean; DVR, direct variable repeat; HGDI, Hunter and Gaston index; IGEPE, infection, genetics, emerging pathogens, evolution; MDR, multidrug resistant; MTC, Mycobacterium tuberculosis complex; $P G G$, principal genetic group; $S$ : N, signal to noise ratio; SNP, single nucleotide polymorphism; TMAC, tetra-methyl ammonium chloride; VNTR, variable number of tandem repeats.
}

A table and figure of spoligotyping data are available as supplementary material with the online version of this paper. relevant to large nations with high TB infection rates, such as Brazil, Russia, India, China and Mexico, but it is also relevant to resource-poor countries in Asia and Africa, which are facing the combined threat of human immunodeficiency virus and TB (Uplekar \& Lonnroth, 2007). TB transmission chains may be interrupted in resource-poor countries by improvements in public health programmes, medical diagnostics facilities and provision of adequate drug treatment (Dye \& Williams, 2008). The Millenium Development Goals of the United Nations Organization has called for the detection of $70 \%$ of active cases through the development of rapid diagnostics methods, and the attainment of $80 \%$ cure rates, through means such as expansion of the Directly Observed Treatment of Short Course $($ DOTS + ) programme. However, the expanding threat of multidrug resistant (MDR) TB (resistance to 
isoniazid and rifampicin) and now of extremely drug resistant (XDR) TB, which is defined as a MDR-TB that is resistant to any fluoroquinolones plus at least one of the three injectable second-line drugs (amikacin, capreomycin, kanamycin) (Manissero \& Fernandez de la Hoz, 2006), has made the implementation of public health measures, and molecular epidemiological investigations using rapid and high throughput molecular methods, a crucial priority to achieve these goals.

TB is caused by the Mycobacterium tuberculosis complex (MTC), which can be defined genetically as a single bacterial species within the order Actinomycetales and encompasses several ecotypes or subspecies that include $M$. tuberculosis, Mycobacterium bovis, Mycobacterium africanum, 'Mycobacterium canettii', M. microti, M. caprae and Mycobacterium pinipedii (Rastogi et al., 2001; Smith et al., 2006). Rapid molecular diagnostics methods have been developed during the two last decades; however, these methods have been slow to be implemented worldwide (Drobniewski et al., 2003). Spoligotyping, comprises a PCR-based reverse line blot hybridization method that assesses the genetic diversity of the direct repeat (DR) locus (Kamerbeek et al., 1997). The DR locus spans up to $5 \mathrm{~kb}$ and represents $0.1 \%$ of the MTC genome. It is a member of the clustered regularly interspaced palindromic repeats (CRISPR) loci genetic family and is found in all Archaea and around $40 \%$ of Eubacteria (Jansen et al., 2002). CRISPR loci are believed to have a functional role against bacteriophage challenge, and at the evolutionary genetics level contain remnants and 'memories' of previous bacteriophage encounters. Due to their genetic polymorphisms, CRISPR loci may be excellent epidemiological markers (Sorek et al., 2008).

The MTC DR locus is made up of a limited number of direct variable repeats (DVRs), each repeat is made up of a conserved 36 bp sequence (the DR) and of a variable 35$41 \mathrm{bp}$ sequence (the spacer). A total of 43 spacers were initially chosen, comprising 6 spacers from M. bovis BCG and 37 spacers from the M. tuberculosis $\mathrm{H} 37 \mathrm{Rv}$ genomes (Groenen et al., 1993; Kamerbeek et al., 1997). Only 10 of these spacers are required to differentiate many of the MTC (Sebban et al., 2002) and this set could be useful as a preliminary 'fast lane' screen. Spoligotyping has been found to be congruent with other molecular techniques, such as multilocus sequence typing (Baker et al., 2004), single nucleotide polymorphism (SNPs) and large sequence polymorphism analysis (Filliol et al., 2006; Gagneux \& Small, 2007; Gutacker et al., 2006). However, for more indepth molecular epidemiological analysis, higher discriminatory genotyping techniques are necessary. These rely on mini-satellite type sequences known as MIRUs (mycobacterial interspaced repeat units) or VNTRs (variable number of tandem repeats). A 24 locus VNTR typing scheme was adopted widely (Supply et al., 2006); it was found to be much more discriminatory than spoligotyping, but not fully concordant, hence there is still a need to perform the two techniques to obtain optimal discrimination. Thus, as spoligotypes are considered both as phylogenetic and epidemiological markers (Asiimwe et al., 2008; Sola et al., 2001; Tanaka \& Francis, 2006; Valcheva et al., 2008), extensive spoligotype databases have proved useful in determining and cataloguing worldwide MTC genetic diversity (Brudey et al., 2006). However, concerns have been raised by some investigators about the potential consequences of suboptimal spoligotyping results, or interpretations of results, on the quality of international databases.

Multiplexing is an efficient way to increase the throughput and efficiency of genotyping results and many recent techniques, such as multiple ligation-mediated assays, are showing promise in this area (Bergval et al., 2008). A DNA chip-based spoligotyping method for the strain identification of $M$. tuberculosis isolates has also been recently developed (Song et al., 2007). All these techniques are particularly well suited to SNP analysis and may be used to assess between 100 to 1000 probes in a single tube (Hardenbol et al., 2003). However, at the lower range of the analysis (50-200 probes), macro-arrays and more recently the suspension microarray format are proving sufficiently powerful and economical genotyping methods (Kaufhold et al., 1994; Goguet de la Salmoniere et al., 2004; Dunbar et al., 2003).

A single study by the Centers for Disease Control and Prevention, USA, evaluated the possibility of transferring spoligotyping to a multiplex microbead-based suspension array format (Cowan et al., 2004). However, economical restrictions have meant that this has not yet been widely implemented. Yet as the cost of medical diagnosis devices drop, and newer and even more innovative systems will be developed, this versatile microbead suspension format could prove useful even in resource-limited settings because: (i) it is a simple and powerful multianalyte system, (ii) the use of the microspheres is easy with a 96well microplate format, (iii) it is flexible and has the opportunity to include new or customized probes, (iv) it has high throughput, (v) it is a time-saving technique, (vi) it increases the quality of results. Such a molecular diagnostic and genotyping device could be fully dedicated to public health-oriented MTC genotyping projects and would provide effective support for epidemiological studies in national TB reference centres, and other TB diagnostic laboratories that have the capacity for mycobacterial cultures and DNA preparation.

In this article, we describe the implementation of and improvements to the spoligotyping technique in different formats, including suggestions for an increased discrimination format or simplifications to create a reduced costeffective form suitable for a wide range of settings.

\section{METHODS}

Chemicals, buffers, microbeads and oligonucleotides. $\mathrm{N}$-(3dimethylaminopropyl)- $N$-ethylcarbodiimide (EDC) was used for 
oligonucleotide-microbead coupling (Pierce Chemical) in 0.1 M MES buffer solution, pH 4.5 (Sigma-Aldrich). Hybridization solutions of $1.5 \times$ and $1 \times$ TMAC (tetra-methyl ammonium chloride) were prepared using 5 M TMAC (Sigma-Aldrich), $20 \%$ sarkosyl (sodium lauroylsarcosinate), Tris/ $\mathrm{HCl}$ and $\mathrm{EDTA}$, and were prepared as described by Cowan et al. (2004). Oligonucleotides (25mers) were modified (van Embden et al., 2000) from described sequences (Kamerbeek et al., 1997) (Table 1). Panel 1 used a standardized 43 spacer format (Sebban et al., 2002). Panel 2 (25 more spacers) was chosen to improve the discrimination for the MTC M. africanum subspecies and for the East African-Indian (EAI) clade (van Embden et al., 2000). The 10 spacers of panel 3 included spacer number 7, 9, $18,22,29,31,32,33,34$ and 43 (Kamerbeek nomenclature), and were chosen based on previous bioinformatics results (Sebban et al., 2002). Primers 5'-biotinylated DRa (GGTTTTGGGTCTGACGAC) and DRb (CCGAGAGGGGACGGAAAC) were used for the spoligotyping PCR. All capture oligonucleotides (Eurogentec) were supplied with a $5^{\prime}$ terminal amino group modification using a 12 carbon spacer linker to increase the free space between the microspheres and the oligonucleotides for a better hybridization.

Microbead coupling. Luminex xMap microbeads (L100-C101 to L100-C168) were resuspended by vortexing (20 s) and sonication (20 s). Microbeads (2.5 million) were transferred to an Eppendorf Protein LoBind tube and pelleted at $8000 \mathrm{~g}$ for $1-2 \mathrm{~min}$. The supernatant was discarded and the pellet was resuspended in $25 \mu \mathrm{l}$ $0.1 \mathrm{M}$ MES buffer $(\mathrm{pH}=5.4)$. Oligonucleotides were suspended in ultrapure water at $1 \mathrm{mM}$ and $100 \mathrm{pmol}$ oligonucleotide capture probe previously diluted to $0.1 \mathrm{mM}$ was added to the microbeads. A fresh EDC solution $\left(10 \mathrm{mg} \mathrm{ml}^{-1}\right)$ was prepared and $1.25 \mu \mathrm{l}$ was added to each Eppendorf tube. Incubation was carried out in the dark at room temperature for $30 \mathrm{~min}$. Subsequently $1.25 \mu \mathrm{l}$ EDC was added to each tube and incubation at room temperature was carried out in the dark for a further $30 \mathrm{~min}$. The microbeads were then washed with $0.5 \mathrm{ml}$ Tween $20(0.02 \%)$ followed by $0.5 \mathrm{ml}$ SDS $(0.1 \%)$, and then resuspended in $50 \mu \mathrm{l}(1 \times) \mathrm{TE}(10 \mathrm{mM}$ Tris, $1 \mathrm{mM}$ EDTA, pH 8) with vortexing and sonication. Coupled microbeads were stored in the dark at $4{ }^{\circ} \mathrm{C}$ until required. A mixture of appropriate beads, depending on the assay, was prepared by combining equal volumes of each selected coupled set. Microbead concentration was checked by haemocytometer. Bead coupling efficiency was checked for the first designed capture probe using hybridization with a biotinylated-antisense detection probe followed by the multianalyte dual-laser detection.

Spoligotyping PCR protocol. PCR ( $25 \mu$ total) was performed for 20 cycles (Kamerbeek et al., 1997) using thermolysates as samples. This was expected to generate $10^{6}$ amplification copies of each spacer for each tube.

Microbead-based multianalyte two-laser system: principle, hardware and software. The system (Luminex 200) is made up of three components: an analyzer/reader, a microplate handler and a sheath-fluid delivery component. The software used was Luminex version 2.3. The device uses oligonucleotide-coupled microspheres (diameter $5.4 \mu \mathrm{m}$ ) as support, with oligonucleotide capture probes that have been previously covalently linked to the microspheres. If, within a PCR product, the target of the capture probe is present, it will hybridize to the microbead surface. Each set of microbeads is characterized by a combination of two dyes (one red and one near infrared) so that each set has a unique spectral address. Beads are presented individually to detection lasers through a quartz cuvette. A $635 \mathrm{~nm}$ red laser excites the two dyes in each bead and identifies which set it belongs to. A $532 \mathrm{~nm}$ green laser is the reporter laser that excites the fluorochromes bound to the PCR product hybridized to the beads and provides quantification.
Hybridization. A defined microsphere mixture was prepared by addition of each set of stocked beads in $1.5 \times$ TMAC hybridization buffer to a final concentration of approximately 150 microspheres $\mu^{-1}$ for each set. For hybridization, we added $2 \mu \mathrm{l}$ PCR product into $33 \mu \mathrm{l}$ microsphere mixture. After a denaturation at $95{ }^{\circ} \mathrm{C}$ for $5 \mathrm{~min}$, incubation was done at $52{ }^{\circ} \mathrm{C}$ for $15 \mathrm{~min}$. Plates were centrifuged at $2250 \mathrm{~g}$ for $3 \mathrm{~min}$ and half of the supernatant ( $30 \mu$ approximately) was replaced with the same amount of $1 \times$ TE. A reporter mix $(25 \mu \mathrm{l})$ was prepared by adding $4 \mu \mathrm{l}$ streptavidin-R-phycoerythrin (1 mg $\mathrm{ml}^{-1}$ ) (Molecular Probes) to $996 \mu \mathrm{l} 1 \times$ TMAC hybridization buffer in the plates. After a final incubation again at $52{ }^{\circ} \mathrm{C}$ for $5 \mathrm{~min}$, mixtures were analysed by the microplate reader as suggested in the user's manual.

Data analysis. The distribution of the values obtained for each spacer was determined, as described by Cowan et al. (2004), as bimodal. A cut-off value was initially chosen by eye for each spacer. A mean negative value and mean positive value were then calculated and the signal to noise ratio $(\mathrm{S}: \mathrm{N})$ derived from the ratio of the two means. We also extrapolated a mean control value and derived a simple calculation linking the cut-off value and the mean negatives.

Statistical analysis. The Hunter and Gaston index (HGDI) was computed to show the superiority of the 68 spacers versus 43 spacers technique for the EAI and M. bovis clades (Hunter \& Gaston, 1988).

Mycobacterial isolates, origin, DNA extraction. Two independent sets of 50 DNA extractions were obtained from MTC clinical isolates from the Bichat Claude Bernard Hospital, Paris (R. Ruimy, unpublished results). Both sets had been previously genotyped by spoligotyping using commercial membranes (Isogen Biosciences). One set was representative of the overall genetic diversity of $M$. tuberculosis found in Paris; the second was selected to include more $M$. africanum and EAI isolates, and other less defined principal genetic group (PGG) 1 and PGG 2 organisms (Sreevatsan et al., 1997) based on their spoligotyping signatures determined by Soini et al. (2000). A further set of DNA samples extracted from a set of bovine clinical isolates from the French Food Safety Agency Laboratory for bovine tuberculosis (Agence Française de Sécurité Sanitaire des Aliments, Maisons-Alfort, France) $(n=234)$ and a last set obtained from human clinical isolates from the Institut Pasteur du Cambodge $(n=118)$ were also tested using the 68 spacer set format. Ten more DNA samples extracted from as many clinical isolates from a previous molecular epidemiological study performed in Albania (Tafaj et al., 2009) were also included on a reduced spacer panel (panel 3). All DNAs were extracted using a thermolysate method (three cycles of freeze-thaw followed by centrifugation and removal of cell pellets). DNA samples were stored at $-20{ }^{\circ} \mathrm{C}$ until use.

\section{RESULTS AND DISCUSSION}

\section{Development of the classical 43 spacers format assay (panel 1)}

We initially tested the microbead-based multianalyte duallaser device with a standard spoligotyping format by coupling 43 oligonucleotide spacers to microbeads. A total of 50 MTC DNAs from clinical isolates obtained from Bichat Claude Bernard Hospital, Paris, were blinded and genotyped using both microbead-based and membranebased formats (Table 1). In the microbead format the distribution value of all experimental relative fluorescence intensity (RFI) data points showed an absence of overlap between negative and positive RFI values, independent of 
Table 1. List of spacer oligonucleotide sequences of MTC used in this study (capture probes)

\begin{tabular}{|c|c|c|}
\hline Genome spacer no. & 43 spacer format no. & Oligonucleotide sequence $\left(5^{\prime} \rightarrow 3^{\prime}\right)$ \\
\hline 1 & & ATAGAGGGTCGCCGGCTCTGGATCA \\
\hline 2 & 1 & CCTCATGCTTGGGCGACAGCTTTTG \\
\hline 3 & 2 & CCGTGCTTCCAGTGATCGCCTTCTA \\
\hline 4 & 3 & ACGTCATACGCCGACCAATCATCAG \\
\hline 5 & & TTTTCTGACCACTTGTGCGGGATTA \\
\hline 6 & & CGTCGTCATTTCCGGCTTCAATTTC \\
\hline 7 & & GAGGAGAGCGAGTACTCGGGGCTGC \\
\hline 8 & & CGTGAAACCGCCCCCAGCCTCGCCG \\
\hline 9 & & ACTCGGAATCCCATGTGCTGACAGC \\
\hline 10 & & TCGACACCCGCTCTAGTTGACTTCC \\
\hline 11 & & GTGAGCAACGGCGGCGGCAACCTGG \\
\hline 12 & 4 & ATATCTGCTGCCCGCCCGGGGAGAT \\
\hline 13 & 5 & GACCATCATTGCСАТTСССТСТССС \\
\hline 14 & 6 & GGTGTGATGCGGATGGTCGGCTCGG \\
\hline 15 & 7 & CTTGAATAACGCGCAGTGAATTTCG \\
\hline 16 & & CGAGTTCCCGTCAGCGTCGTAAATC \\
\hline 17 & & GCGCCGGCCCGCGCGGATGACTCCG \\
\hline 18 & 8 & CATGGACCCGGGCGAGCTGCAGATG \\
\hline 19 & 9 & TAACTGGCTTGGCGCTGATCCTGGT \\
\hline 20 & 10 & TTGACCTCGCCAGGAGAGAAGATCA \\
\hline 21 & 11 & TCGATGTCGATGTCCCAATCGTCGA \\
\hline 22 & 12 & ACCGCAGACGGCACGATTGAGACAA \\
\hline 23 & 13 & AGCATCGCTGATGCGGTCCAGCTCG \\
\hline 24 & 14 & CCGCCTGCTGGGTGAGACGTGCTCG \\
\hline 25 & 15 & GATCAGCGACCACCGCACCCTGTCA \\
\hline 26 & 16 & CTTCAGCACCACCATCATCCGGCGC \\
\hline 27 & 17 & GGATTCGTGATCTCTTCCCGCGGAT \\
\hline 28 & 18 & TGCCCCGGCGTTTAGCGATCACAAC \\
\hline 29 & 19 & AAATACAGGCTCCACGACACGACCA \\
\hline 30 & 20 & GGTTGCCCCGCGCCCTTTTCCAGCC \\
\hline 31 & 21 & TCAGACAGGTTCGCGTCGATCAAGT \\
\hline 32 & 22 & GACCAAATAGGTATCGGCGTGTTCA \\
\hline 33 & 23 & GACATGACGGCGGTGCCGCACTTGA \\
\hline 34 & 24 & AAGTCACCTCGCCCACACCGTCGAA \\
\hline 35 & 25 & TCCGTACGCTCGAAACGCTTCCAAC \\
\hline 36 & 26 & CGAAATCCAGCACCACATCCGCAGC \\
\hline 37 & 27 & CGCGAACTCGTCCACAGTCCCCCTT \\
\hline 38 & 28 & CGTGGATGGCGGATGCGTTGTGCGC \\
\hline 39 & 29 & GACGATGGCCAGTAAATCGGCGTGG \\
\hline 40 & 30 & CGCCATCTGTGCCTCATACAGGTCC \\
\hline 41 & 31 & GGAGCTTTCCGGCTTCTATCAGGTA \\
\hline 42 & 32 & ATGGTGGGACATGGACGAGCGCGAC \\
\hline 43 & 33 & CGCAGAATCGCACCGGGTGCGGGAG \\
\hline 44 & 34 & ATGGCACGGCAGGCGTGGCTAGGGG \\
\hline 45 & & GTGCGCCGTCGCCGTAAGTGCCCCA \\
\hline 46 & 35 & TTTCGACGACAATTCGTTGACCACG \\
\hline 47 & 36 & GTTACCGCTGGCGCGCATCATTCAT \\
\hline 48 & & CGTGCACATGCCGTGGCTCAGGGGT \\
\hline 49 & & CATGCAGCATGCCGTCCCCGTTTTT \\
\hline 50 & & TGCTCTTGAGCAACGCCATCATCCG \\
\hline 51 & 37 & GGCAAGTTGGCGCTGGGGTCTGAGT \\
\hline 52 & 38 & GCGAGGAACCGTCCCACCTGGGCCT \\
\hline 53 & 39 & GGAAACGCAGCACCAGCCTGACAAT \\
\hline 54 & & GCACTGCAACCCGGAATTCTTGCAC \\
\hline 55 & & CCATATCGGGGACGGCGACGCTGCG \\
\hline 56 & & ACGCGTCGTGCCATCAGTCAGCGTC \\
\hline
\end{tabular}


Table 1. cont.

\begin{tabular}{|c|c|c|}
\hline Genome spacer no. & 43 spacer format no. & Oligonucleotide sequence $\left(5^{\prime} \rightarrow 3^{\prime}\right)$ \\
\hline 57 & & AACACTTTTTTTGAGCGTGGCGCGG \\
\hline 58 & & GGGCATCGATCATGAGAGTTGCGTT \\
\hline 59 & & CTGGCGACGATTTTCGCTGTTGTGG \\
\hline 60 & & AGCACCTCCCTTGACAATCCGGCAG \\
\hline 61 & & GGCCTAAGGGTGCTGACTTCGCCTG \\
\hline 62 & 40 & ACGACGAGCAGCGGCATACAGAGCC \\
\hline 63 & 41 & TTGCATCCACTCGTCGCCGACACGG \\
\hline 64 & 42 & TGGTAATTGCGTCACGGCGCGCCTG \\
\hline 65 & 43 & ACCATCCGACGCAGGCACCGAAGTC \\
\hline 66 & & CACACCACAGCCACGCTACTGCTCC \\
\hline 67 & & ACACCGCCGATGACAGCTATGTCCG \\
\hline 68 & & CTTCGCGCGGTGTTTCGGCCGTGCC \\
\hline
\end{tabular}

the probe detected. Negative RFI values varied between spacers and were significantly high for spacer 29 and low for spacer 15 . We observed only $2 / 2150$ discrepant data points among 50 samples tested between the two techniques. These were linked to spacer 15 for the samples 2007-2 and 2007-13, and called negative by membranebased and positive by microbead-based spoligotyping. As the values of spacer 15 were above the cut-off value for the microbead-based assay, we concluded that for these samples the spacer 15 was present. A repeat run using a fresh membrane confirmed the presence of the spacer 15 in the two samples. These discrepant results were not wholly unexpected as this spacer has previously been shown to give a low reproducibility in membrane-based methods probably as a result of genomic structural configurations (van Embden et al., 2000). Indeed, this problem could possibly be explained by a published study showing that the DVR26 [van Embden et al. (2000) numbering, or DR16 in the Kamerbeek et al. (1997) numbering] contains a 4 bp deletion in the adjacent DR (van Embden et al., 2000) and that the deletion in DR16 could decrease the relative amount of spacer 15 amplification, which ultimately lead to a lower $\mathrm{S}: \mathrm{N}$ ratio. Nevertheless, despite the lower signal we could clearly distinguish between positive and negative values confirming the power of the microbead-based technique. We also observed a low $\mathrm{S}: \mathrm{N}$ for spacer 15 on beads as previously seen in membrane-based assays. The addition of a washing step increased $\mathrm{S}: \mathrm{N}$ for all spacers (see experiment 2 compared to experiment 1 in Table 2). Additional typing experiments confirmed that the cut-off value remained constant with each set of beads and that the method was fully reproducible (H37Rv and M. bovis BCG, and a negative control, were systematically included).

Obtaining good results using the classical membrane-based spoligotyping assay critically depends on the quality of a membrane. Although theoretically reusable up to $10-20$ times, commercial membranes tend to provide lesser quality results after 6-8 uses and this phenomenon was responsible of some of the discrepancies we observed initially. In this work, these instances were few and were all solved after retesting using a new membrane. It is also clear that coupling efficiency (i.e. between the beads and the probes) may also influence the quality of the results. However, we did not experience problems in this regard. We nevertheless advise recalculation of cut-off values for individual batches of coupled beads.

RFI values of all spacers for some samples were lower than those obtained for other samples. This result was most likely caused by the use of inferior quality DNA leading to suboptimal PCR amplification as in this study we used DNA extracted from thermolysates, which are known to often generate low-quality samples. Yet even using these suboptimal extractions the discriminative power resulting from this procedure was high, suggesting that even with unpurified (low DNA concentration) thermolysates the method can be considered as highly robust. Recent results on a much larger set of samples (more than 4000) showed that cetyl-trimethylammonium-extracted DNA gave higher $\mathrm{S}: \mathrm{N}$ than thermolysate DNA, further simplifying analysis (data not shown).

\section{Extended spoligotyping using $\mathbf{2 5}$ more spacers (panel 2)}

A subset of PGG 1 isolates, as defined by Sreevatsan et al. (1997) and Soini et al. (2000), from the first set of 50 DNAs, combined with a further set that included a majority of PGG 1 clinical isolates ( $n=402$ total), was chosen to test an improved spoligotyping format. Indeed, published studies have suggested that PGG 1 spoligotyping could be improved by the addition of spacers; this is not the case with 'Haarlem' clinical isolates (PGG 2) (van Embden et al., 2000). Positive and negative values could be clearly distinguished without overlap, with the apparent exception of spacer 48 for which five isolates belonging to the Central Asian (CAS) clade provided trimodal signals (Supplementary Fig. S1 available with the online journal). This observation suggested intermediate hybridization signals; however, sequencing did not confirm the presence of this spacer. This result remains for the time-being 
Table 2. $\mathrm{S}: \mathrm{N}$ in microbead-based spoligotyping

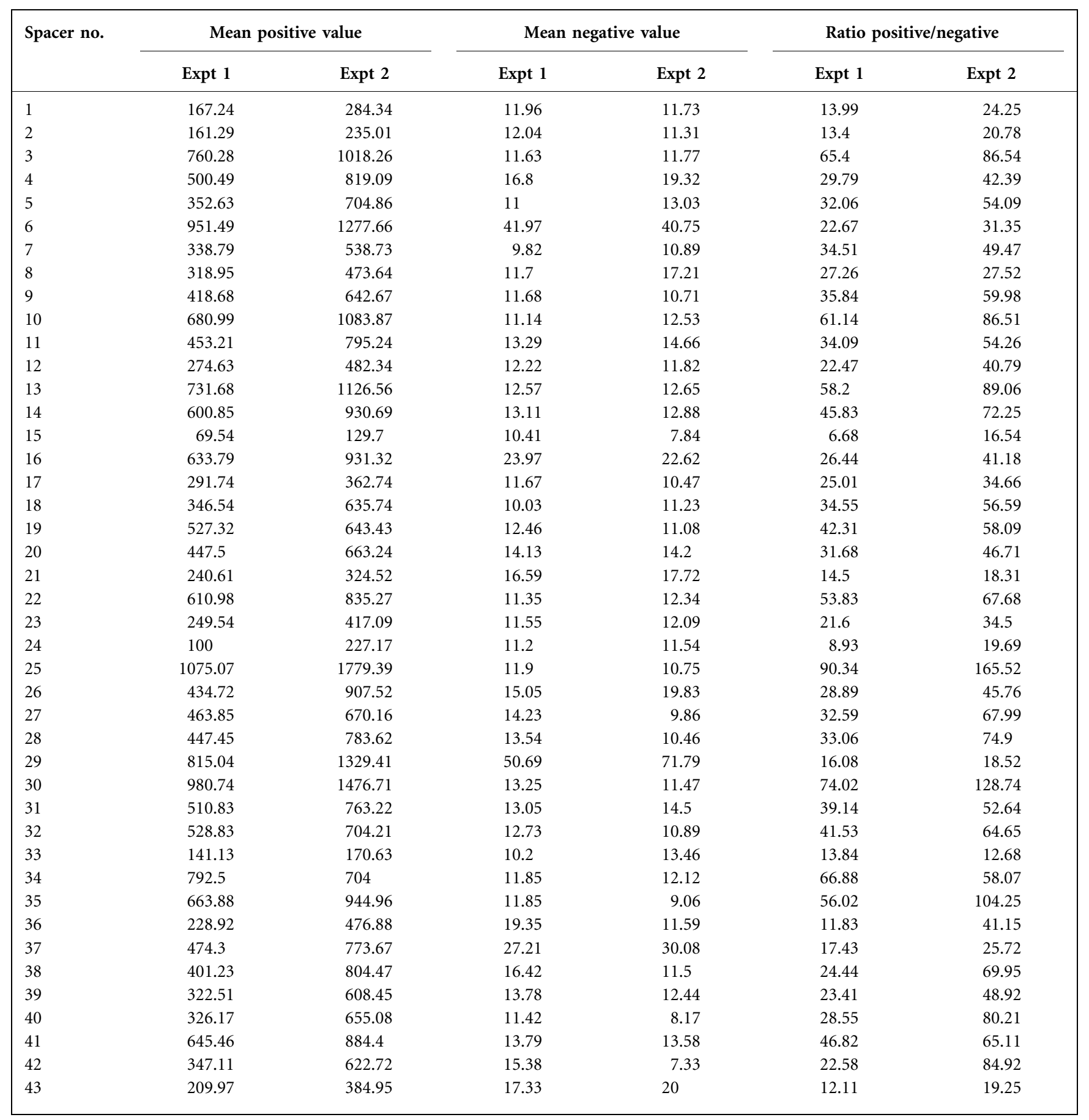

unexplained. Among the 402 DNAs assessed using the 68 spacer spoligotyping, 351 DNAs from essentially two new origins (Agence Française de Sécurité Sanitaire des Aliments for M. bovis and Institut Pasteur du Cambodge for the EAI clade) were clustered into seven clades [M. africanum, M. bovis, Beijing, EAI, CAS, Latin-American and Mediterranean (LAM) and $\mathrm{T}$ (all other modern isolates)]. Results provided a greater discrimination of $M$. africanum, EAI and M. bovis than had been described by Sreevatsan et al. (1997). The most significant results are shown in Table 3 (clustering results for 351 clinical isolates) and in Supplementary Table S1 (available with the online journal) (unique patterns description). A computation of the HGDI on the samples belonging to the M. bovis and to the EAI clades, when using the 68 versus 43 spacers format, shows an increase of HGDI from 0.83 to 0.85 (for M. bovis) and from 0.93 to 0.96 (for EAI). According to the current available requirements (HGDI $>0.95$ for a molecular marker to be used in epidemiology), spoligotyping under the 68 spacers format would then 
Table 3. Comparison of cluster number obtained with the 43 spacers format as compared with the 68 spacers format

\begin{tabular}{|lccc|}
\hline & \multirow{2}{*}{ No. of isolates } & \multicolumn{2}{c|}{ No. of clusters } \\
\cline { 3 - 4 } & & $\mathbf{4 3}$ format & $\mathbf{6 8}$ format \\
\hline M. africanum & 13 & 3 & 5 \\
M. bovis & 224 & 13 & 19 \\
EAI & 63 & 12 & 21 \\
Beijing & 30 & 1 & 2 \\
CAS & 7 & 1 & 2 \\
LAM & 8 & 1 & 1 \\
T & 6 & 2 & 2 \\
Total & 351 & 33 & 52 \\
\end{tabular}

$\mathrm{T}$, Modern isolates (all other undifferentiated isolates).

become a suitable method to run population-based molecular epidemiological studies in South-East Asia where the EAI clade is prevalent. Our sampling on $M$. africanum is currently too low to conclude on the superiority of the 68 versus 43 spoligotyping format.

The introduction of 25 additional spacers, derived from $M$. bovis and M. tuberculosis sequences (Beggs et al., 1996), provided the greatest discriminatory power so far achieved within the MTC complex, with the exception of the ' $M$. canettii subspecies whose typing would require 26 more spacers (van Embden et al., 2000). In previous works, we and other investigators have suggested that the introduction of 25 new spacers could improve genotyping, especially for large animal and human-adapted MTC PGG 1 subspecies (Brudey et al., 2004; van der Zanden et al., 2002; Javed et al., 2007). In this study, we confirm that this improvement is significant for the study of the clinical isolates belonging to the EAI clade that is prevalent in South-East Asia.

\section{Development of a simplified 10 spacers format assay (panel 3)}

Other genetic diversity studies on MTC using IS6110RFLP, VNTR, SNPs or region of differences diversity studies have identified a phylogeographical population structure in this monomorphic bacterial species (Baker et al., 2004; Dos Vultos et al., 2008; Gagneux \& Small, 2007; Gutacker et al., 2006; Kremer et al., 1999; Sola et al., 2003; van Embden et al., 1993). A previous bioinformatics study has shown that a limited set of spoligotype spacers $(n=10$, spacers number $7,9,18,22,29,31,32,33,34$ and 43) could be sufficient to discriminate between the most prevalent MTC phylogeographical types (Sebban et al., 2002). Using these spacers we also implemented a simplified microbeadbased spoligotyping assay format, which could be used as a relatively inexpensive 'first screen' genotyping technique for MTC. This assay could also be used as an external quality control for laboratories still working with mem- brane-based spoligotyping, or as a first step toward further multiplexing. Other markers such as rifampicin-resistance mutations $(r p o B)$ could be included in these assays. The use of a 10 spacers assay (panel 3 ) would naturally be cheaper, and still remain an informative assay that could be suited to resource-limited countries. Improving the capacity and transfer of rapid molecular diagnostics methods, whilst decreasing prices of emerging technologies, are undoubtedly desirable objectives towards good public-health strategies and achieving a more sustainable decrease in preventable deaths caused by poverty-related infectious diseases.

\section{Comparison of the cost/output ratio of the microsphere-based versus the membrane-based assay for spoligotyping}

Spoligotyping, either using a microbead-based multianalyte genotyping platform or membrane-based, is a rapid diagnostic test most suitable for studying TB disease in high-burden countries. One important parameter for technology transfer, especially for resource-poor or middle-income countries, will be the cost/output ratio needed to introduce the new technique (microbead-based high throughput) compared to the established one (membranebased low throughput). We thus evaluated the relative costs of material, reagents and staff required to genotype 5000 samples by the two techniques (based on a 'standardized' 43 spacers format). The results (Table 4) showed that running spoligotyping through a high throughput multianalyte hybridization device would be $30-40 \%$ more expensive than a standard membrane-based technique. The throughput is at least $4-8$ times higher using the microbead-based rather than the membranebased approach. It also improves the global quality in TB genotyping by (i) introducing standardized cut-off values thus improving standardization of spoligotyping, (ii) theoretically helping to discover new SNPs in CRISPR loci, (iii) increasing the potential use of newly discovered spacers (Brudey et al., 2004; van der Zanden et al., 2002; van Embden et al., 2000). In countries where TB outbreaks are largely linked to $M$. africanum, the EAI clade or even the CAS clade, i.e. within Africa and the Indian subcontinent and Asia (Ahmed \& Leblebicioglu, 2006), this is particularly relevant.

The microbead-based format allows a digital-numerical data output less prone to interpretation errors. This output is also more amenable to being managed through large international databases that can be curated for genotyping errors.

The advent of molecular epidemiological techniques using polymorphic repeated DNA markers has improved ways to investigate TB outbreaks (van Soolingen, 2001). In particular they have been used to demonstrate to clinicians, epidemiologists and public health investigators that recent TB transmission rates were higher than previously estimated (Alland et al., 1994), concomitantly to the decision by the World Health Organization in 1994 to 
Table 4. Economic analysis of automated versus manual spoligotyping

\begin{tabular}{|c|c|c|}
\hline Parameter & Multiplexed microbead-based (automated) & Membrane-based (manual) \\
\hline Device cost & Around 60000 Euros (Luminex 200) & $\begin{array}{l}1200 \text { Euros (Miniblotter, } \\
\text { Immunetics) }\end{array}$ \\
\hline $\begin{array}{l}\text { Spoligotyping kits (5000 detection) (Ocimum } \\
\text { Biosolutions) }\end{array}$ & - & 3300 Euros \\
\hline $\begin{array}{l}\text { Full set of } 43 \text { microbeads (5000 detection), } \\
\text { control and calibration }\end{array}$ & 8600 Euros & - \\
\hline Oligonucleotides costs & 1200 Euros & None \\
\hline PCR product volume required & $5 \mu \mathrm{l}$ & $25 \mu \mathrm{l}$ \\
\hline Working time for 500 analyses & 2 days & 11 days \\
\hline Flexibility & Yes & More or less \\
\hline Operator dependent & $\begin{array}{l}\text { No; however, needs a well trained } \\
\text { technician }\end{array}$ & $\begin{array}{l}\text { Yes, needs a carefully trained } \\
\text { technician }\end{array}$ \\
\hline Result interpretation & No (automated) & Yes \\
\hline Database comparison required & Yes & $\begin{array}{l}\text { Yes for full usefulness of information } \\
\text { produced }\end{array}$ \\
\hline Open or closed system & Open & $\begin{array}{l}\text { Open (investigator may choose to } \\
\text { build its own kit) }\end{array}$ \\
\hline Development & Yes (could be applied to new SNPs) & $\begin{array}{l}\text { Yes (other probes and targets can be } \\
\text { tested) }\end{array}$ \\
\hline Possibility of improved technique & Yes & Yes \\
\hline $\begin{array}{l}\text { Data production technician time for theoretical } \\
5000 \text { assays }\end{array}$ & 25 days & 110 days \\
\hline $\begin{array}{l}\text { Data reading and control technician time for } \\
5000 \text { assays }\end{array}$ & $25 \mathrm{~h}$ & $230 \mathrm{~h}$ \\
\hline Total working costs (time) & $200 \mathrm{~h}$ & $1000 \mathrm{~h}$ \\
\hline $\begin{array}{l}\text { Total working costs (in Euros) if unitary } \\
\text { working cost is set to } 50 \text { Euros } \mathrm{h}^{-1}\end{array}$ & 10000 Euros & 50000 Euros \\
\hline Total cost analysis & $80000 / 5000=>16$ Euros $^{*}$ & $55000 / 5000=11$ Euros \\
\hline Pros & $\begin{array}{l}\text { High throughput, results generated } \\
\text { directly in Excel format. Ideally suited } \\
\text { for large centralized analysis production } \\
\text { facilities in new emerging high-burden } \\
\text { countries like Brazil, Russia, India, } \\
\text { China. Can be linked to a LIMS }\end{array}$ & $\begin{array}{l}\text { Robust, simple technology; however, } \\
\text { lot of error sources, results are } \\
\text { interpretative and require expertise, } \\
\text { spacer } 15 \text { doubtful }\end{array}$ \\
\hline Cons & $\begin{array}{l}\text { More expensive at present, should be } \\
\text { done in limited number of level } 3 \text { or } 4 \\
\text { mycobacteriology laboratories }\end{array}$ & $\begin{array}{l}\text { Less discriminative than MLVA } \\
\text { (multilocus VNTR analysis) }\end{array}$ \\
\hline
\end{tabular}

LIMS, Laboratory Information Management System.

${ }^{\star}$ Cost of 43 spacers-based technology.

declare TB a global emergency (Nakajima, 1993). The recent global expansion of MDR-TB and XDR-TB has renewed calls for new public health-oriented research programmes (Dye \& Williams, 2008) and the introduction of more efficient and economical genotyping methods worldwide in mycobacteriology laboratories.

High-throughput multiplexed hybridization techniques are being increasingly developed for molecular diagnostics, molecular epidemiology (Dunbar et al., 2003; Jiang et al., 2006) and environmental studies (Baums et al., 2007). The recent transfer of the 'spoligotyping' technique to a microbead format (Cowan et al., 2004) provided an opportunity for incorporating this type of assay into our laboratory and promoted a new molecular epidemiological study. Indeed the repetitive and heterogeneic nature of CRISPR loci makes them excellent targets for the development of new and powerful genotyping techniques, whilst major diagnostic genomic targets used for bacterial species identification include probes for $23 \mathrm{~S}$ and/or $16 \mathrm{~S}$ rRNA genes (Dunbar, 2006; Mokrousov et al., 2007).

We found the multiplex microbead-based spoligotyping technique to be versatile and easily implemented and it can be adapted (classical 43 spacers format for Europe, improved 68 spacers format for Asia, simplified 10 spacers format for first-screen genotyping in resource-limited settings). It is well established that molecular epidemiolo- 
gical studies in high-prevalence settings are more difficult to interpret and to run than in low-prevalence settings, requiring more analysis of samples and implementation of complex genotyping schemes. This strongly argues in favour of the widespread adoption of high-throughput methods (Hanekom et al., 2008).

Further developments in this system will include study of other epidemiologically important clades (such as Beijing) and development of links to SNP-based typing methods allowing clinical, phylogenetic and epidemiological improvements in rapid molecular methods with the aim of providing simultaneous strain typing and molecular antibiogrammes to further aid in control regimens aimed against MDR and XDR-TB.

In conclusion the suspension microarray spoligotyping assay was efficiently implemented, improved and represents a viable and practical alternative to MIRU-VNTR as a first screen for large population-based systematic genotyping programs. An international multicentred project is currently ongoing to confirm our results on a larger scale.

\section{ACKNOWLEDGEMENTS}

This work was made possible thanks to a research grant from the Fondation bioMérieux, Lyon, France, and Dr Guy Vernet is warmly acknowledged. M. François Topin (Luminex Europe, Netherlands) is warmly acknowledged for his support. The IGEPE team was created thanks to the University Paris-Sud 11 and to the Director of the Institut of Genetics and Microbiology (IGM) (UMR8621), Dr Monique Bolotin-Fukuhara. J.Z. holds a $\mathrm{PhD}$ fellowship from the Presidency of the University of Paris-Sud 11. Dr Gilles Vergnaud, Head of the GPMS Research Team at IGM, is also warmly acknowledged for his help. Lastly, we are grateful to Professor B. Gicquel, Head of the Mycobacterial Genetics Unit at the Institut Pasteur, for her permanent support and helpful discussions.

\section{REFERENCES}

Ahmed, N. \& Leblebicioglu, H. (2006). India's 'gold mine' of ancestral bacilli and the looming TB-HIV pandemic. Ann Clin Microbiol Antimicrob 5, 31.

Alland, D., Kalkut, G. E., Moss, A. R., Adam, R. A. M., Hahn, J. A., Bosworth, W., Drucker, E. \& Bloom, B. R. (1994). Transmission of tuberculosis in New York City: an analysis by DNA fingerprinting and conventional epidemiologic methods. N Engl J Med 330, 1710-1716.

Asiimwe, B. B., Koivula, T., Kallenius, G., Huard, R. C., Ghebremichael, S., Asiimwe, J. \& Joloba, M. L. (2008). Mycobacterium tuberculosis Uganda genotype is the predominant cause of TB in Kampala, Uganda. Int J Tuberc Lung Dis 12, 386-391.

Baker, L., Brown, T., Maiden, M. C. \& Drobniewski, F. (2004). Silent nucleotide polymorphisms and a phylogeny for Mycobacterium tuberculosis. Emerg Infect Dis 10, 1568-1577.

Baums, I. B., Goodwin, K. D., Kiesling, T., Wanless, D., Diaz, M. R. \& Fell, J. W. (2007). Luminex detection of fecal indicators in river samples, marine recreational water, and beach sand. Mar Pollut Bull 54, 521-536.

Beggs, M. L., Cave, M. D., Marlowe, C., Cloney, L., Duck, P. \& Eisenach, K. D. (1996). Characterization of Mycobacterium tuber- culosis complex direct repeat sequence for use in cycling probe reaction. J Clin Microbiol 34, 2985-2989.

Bergval, I. L., Vijzelaar, R. N., Dalla Costa, E. R., Schuitema, A. R., Oskam, L., Kritski, A. L., Klatser, P. R. \& Anthony, R. M. (2008). Development of multiplex assay for rapid characterization of Mycobacterium tuberculosis. J Clin Microbiol 46, 689-699.

Brudey, K., Gutierrez, M. C., Vincent, V., Parsons, L. M., Salfinger, M., Rastogi, N. \& Sola, C. (2004). Mycobacterium africanum genotyping using novel spacer oligonucleotides in the direct repeat locus. J Clin Microbiol 42, 5053-5057.

Brudey, K., Driscoll, J., Rigouts, L., Prodinger, W. M., Gori, A., Al-Hajoj, S. A. M., Allix, C., Aristimuno, L., Arora, J. \& other authors (2006). Mycobacterium tuberculosis complex genetic diversity: mining the fourth international spoligotyping database (SpolDB4) for classification, population genetics, and epidemiology. $B M C$ Microbiol 6, 23.

Cowan, L. S., Diem, L., Brake, M. C. \& Crawford, J. T. (2004). Transfer of a Mycobacterium tuberculosis genotyping method, spoligotyping, from a reverse line-blot hybridization, membrane-based assay to the Luminex multianalyte profiling system. J Clin Microbiol 42, 474-477.

Dos Vultos, T., Mestre, O., Rauzier, J., Golec, M., Rastogi, N., Rasolofo, V., Tonjum, T., Sola, C., Matic, I. \& other authors (2008). Evolution and diversity of clonal bacteria: the paradigm of Mycobacterium tuberculosis. PLoS One 3, e1538.

Drobniewski, F. A., Caws, M., Gibson, A. \& Young, D. (2003). Modern laboratory diagnosis of tuberculosis. Lancet Infect Dis 3, 141-147.

Dunbar, S. A. (2006). Applications of Luminex xMAP technology for rapid, high-throughput multiplexed nucleic acid detection. Clin Chim Acta 363, 71-82.

Dunbar, S. A., Vander Zee, C. A., Oliver, K. G., Karem, K. L. \& Jacobson, J. W. (2003). Quantitative, multiplexed detection of bacterial pathogens: DNA and protein applications of the Luminex LabMAP system. J Microbiol Methods 53, 245-252.

Dye, C. \& Williams, B. G. (2008). Eliminating human tuberculosis in the twenty-first century. $J R$ Soc Interface 5, 653-662.

Filliol, I., Motiwala, A. S., Cavatore, M., Qi, W., Hernando Hazbon, M., Bobadilla Del Valle, M., Fyfe, J., Garcia-Garcia, L., Rastogi, N. \& other authors (2006). Global phylogeny of Mycobacterium tuberculosis based on single nucleotide polymorphism (SNP) analysis: insights into tuberculosis evolution, phylogenetic accuracy of other DNA fingerprinting systems, and recommendations for a minimal standard SNP set. J Bacteriol 188, 759-772.

Gagneux, S. \& Small, P. M. (2007). Global phylogeography of Mycobacterium tuberculosis and implications for tuberculosis product development. Lancet Infect Dis 7, 328-337.

Goguet de la Salmoniere, Y. O., Kim, C. C., Tsolaki, A. G., Pym, A. S., Siegrist, M. S. \& Small, P. M. (2004). High-throughput method for detecting genomic-deletion polymorphisms. J Clin Microbiol 42, 2913-2918.

Groenen, P. M., Bunschoten, A. E., van Soolingen, D. \& van Embden, J. D. (1993). Nature of DNA polymorphism in the direct repeat cluster of Mycobacterium tuberculosis; application for strain differentiation by a novel typing method. Mol Microbiol 10, 1057-1065.

Gutacker, M. M., Mathema, B., Soini, H., Shashkina, E., Kreiswirth, B. N., Graviss, E. A. \& Musser, J. M. (2006). Single-nucleotide polymorphism-based population genetic analysis of Mycobacterium tuberculosis strains from 4 geographic sites. J Infect Dis 193, 121-128.

Hanekom, M., van der Spuy, G. D., Gey van Pittius, N. C., McEvoy, C. R. E., Hoek, K. G. P., Ndabambi, S. L., Jordan, A. M., Victor, T. C., van Helden, P. D. \& Warren, R. M. (2008). Discordance between mycobacterial interspersed repetitive-unit-variable-number tandemrepeat typing and IS6110 restriction fragment length polymorphism 
genotyping for analysis of Mycobacterium tuberculosis Beijing strains in a setting of high incidence of tuberculosis. J Clin Microbiol 46, $3338-3345$

Hardenbol, P., Baner, J., Jain, M., Nilsson, M., Namsaraev, E. A., Karlin-Neumann, G. A., Fakhrai-Rad, H., Ronaghi, M., Willis, T. D. \& other authors (2003). Multiplexed genotyping with sequence-tagged molecular inversion probes. Nat Biotechnol 21, 673-678.

Hunter, P. R. \& Gaston, M. A. (1988). Numerical index of the discriminatory ability of typing systems: an application of Simpson's index of diversity. J Clin Microbiol 26, 2465-2466.

Jansen, R., van Embden, J. D. A., Gaastra, W. \& Schouls, L. M. (2002). Identification of a novel family of sequence repeats among prokaryotes. OMICS 6, 23-33.

Javed, M. T., Aranaz, A., de Juan, L., Bezos, J., Romero, B., Alvarez, J., Lozano, C., Mateos, A. \& Dominguez, L. (2007). Improvement of spoligotyping with additional spacer sequences for characterization of Mycobacterium bovis and M. caprae isolates from Spain. Tuberculosis (Edinb) 87, 437-445.

Jiang, H. L., Zhu, H. H., Zhou, L. F., Chen, F. \& Chen, Z. (2006). Genotyping of human papillomavirus in cervical lesions by L1 consensus PCR and the Luminex xMAP system. J Med Microbiol 55, 715-720.

Kamerbeek, J., Schouls, L., Kolk, A., van Agterveld, M., van Soolingen, D., Kuijper, S., Bunschoten, A., Molhuizen, H., Shaw, R. \& other authors (1997). Simultaneous detection and strain differentiation of Mycobacterium tuberculosis for diagnosis and epidemiology. J Clin Microbiol 35, 907-914.

Kaufhold, A., Podbielski, A., Baumgarten, G., Blokpoel, M., Top, J. \& Schouls, L. (1994). Rapid typing of group A streptococci by the use of DNA amplification and non-radioactive allele-specific oligonucleotide probes. FEMS Microbiol Lett 119, 19-26.

Kremer, K., van Soolingen, D., Frothingham, R., Haas, W. H., Hermans, P. W., Martin, C., Palittapongarnpim, P., Plikaytis, B. B., Riley, L. W. \& other authors (1999). Comparison of methods based on different molecular epidemiological markers for typing of Mycobacterium tuberculosis complex strains: interlaboratory study of discriminatory power and reproducibility. J Clin Microbiol 37, 26072618.

Manissero, D. \& Fernandez de la Hoz, K. (2006). Surveillance methods and case definition for extensively drug resistant TB (XDRTB) and relevance to Europe: summary update. Euro Surveill 11, E061103.

Mokrousov, I., Limeschenko, E., Vyazovaya, A. \& Narvskaya, O. (2007). Corynebacterium diphtheriae spoligotyping based on combined use of two CRISPR loci. Biotechnol J 2, 901-906.

Nakajima, H. (1993). Tuberculosis: a global emergency. World Health Forum 14, 438.

Rastogi, N., Legrand, E. \& Sola, C. (2001). The mycobacteria: an introduction to nomenclature and pathogenesis. Rev Sci Tech 20, 21-54.

Sebban, M., Mokrousov, I., Rastogi, N. \& Sola, C. (2002). A datamining approach to spacer oligonucleotide typing of Mycobacterium tuberculosis. Bioinformatics 18, 235-243.

Smith, N. H., Kremer, K., Inwald, J., Dale, J., Driscoll, J. R., Gordon, S. V., van Soolingen, D., Glyn Hewinson, R. \& Maynard Smith, J. (2006). Ecotypes of the Mycobacterium tuberculosis complex. J Theor Biol 239, 220-225.

Soini, H., Pan, X., Amin, A., Graviss, E. A., Siddiqui, A. \& Musser, J. M. (2000). Characterization of Mycobacterium tuberculosis isolates from patients in Houston, Texas, by spoligotyping. J Clin Microbiol 38, 669-676.
Sola, C., Filliol, I., Legrand, E., Mokrousov, I. \& Rastogi, N. (2001). Mycobacterium tuberculosis phylogeny reconstruction based on combined numerical analysis with IS1081, IS6110, VNTR and DRbased spoligotyping suggests the existence of two new phylogeographical clades. J Mol Evol 53, 680-689.

Sola, C., Filliol, I., Legrand, E., Lesjean, S., Locht, C., Supply, P. \& Rastogi, N. (2003). Genotyping of the Mycobacterium tuberculosis complex using MIRUs: association with VNTR and spoligotyping for molecular epidemiology and evolutionary genetics. Infect Genet Evol 3, 125-133.

Song, E. J., Jeong, H. J., Lee, S. M., Kim, C. M., Song, E. S., Park, Y. K., Bai, G. H., Lee, E. Y. \& Chang, C. L. (2007). A DNA chip-based spoligotyping method for the strain identification of Mycobacterium tuberculosis isolates. J Microbiol Methods 68, 430-433.

Sorek, R., Kunin, V. \& Hugenholtz, P. (2008). CRISPR - a widespread system that provides acquired resistance against phages in bacteria and archaea. Nat Rev Microbiol 6, 181-186.

Sreevatsan, S., Pan, X., Stockbauer, K. E., Connell, N. D., Kreiswirth, B. N., Whittam, T. S. \& Musser, J. M. (1997). Restricted structural gene polymorphism in the Mycobacterium tuberculosis complex indicates evolutionarily recent global dissemination. Proc Natl Acad Sci U S A 94, 9869-9874.

Supply, P., Allix, C., Lesjean, S., Cardoso-Oelemann, M., RuschGerdes, S., Willery, E., Savine, E., de Haas, P., van Deutekom, H. \& other authors (2006). Proposal for standardization of optimized mycobacterial interspersed repetitive unit-variable-number tandem repeat typing of Mycobacterium tuberculosis. J Clin Microbiol 44, 4498-4510.

Tafaj, S., Zhang, J., Hauck, Y., Pourcel, C., Hafizi, H., Zoraqi, G. \& Sola, C. (2009). First insight into genetic diversity of the Mycobacterium tuberculosis complex in Albania obtained by multilocus variable-number tandem-repeat analysis and spoligotyping reveals the presence of Beijing multidrug-resistant isolates. J Clin Microbiol 47, 1581-1584.

Tanaka, M. M. \& Francis, A. R. (2006). Detecting emerging strains of tuberculosis by using spoligotypes. Proc Natl Acad Sci U S A 103, 15266-15271.

Uplekar, M. \& Lonnroth, K. (2007). MDR and XDR - the price of delaying engagement with all care providers for control of TB and TB/ HIV. Trop Med Int Health 12, 473-474.

Valcheva, V., Mokrousov, I., Rastogi, N., Narvskaya, O. \& Markova, N. (2008). Molecular characterization of Mycobacterium tuberculosis isolates from different regions of Bulgaria. J Clin Microbiol 46, 10141018.

van der Zanden, A. G., Kremer, K., Schouls, L. M., Caimi, K., Cataldi, A., Hulleman, A., Nagelkerke, N. J. \& van Soolingen, D. (2002). Improvement of differentiation and interpretability of spoligotyping for Mycobacterium tuberculosis complex isolates by introduction of new spacer oligonucleotides. J Clin Microbiol 40, 4628-4639.

van Embden, J. D., Cave, M. D., Crawford, J. T., Dale, J. W., Eisenach, K. D., Gicquel, B., Hermans, P., Martin, C., McAdam, R. \& other authors (1993). Strain identification of Mycobacterium tuberculosis by DNA fingerprinting: recommendations for a standardized methodology. J Clin Microbiol 31, 406-409.

van Embden, J. D. A., van Gorkom, T., Kremer, K., Jansen, R., van der Zeijst, B. A. M. \& Schouls, L. M. (2000). Genetic variation and evolutionary origin of the direct repeat locus of Mycobacterium tuberculosis complex bacteria. J Bacteriol 182, 2393-2401.

van Soolingen, D. (2001). Molecular epidemiology of tuberculosis and other mycobacterial infections: main methodologies and achievements. J Intern Med 249, 1-26. 